\title{
Диагностический и лечебный процесс в психиатрии и психотерапии - единый процесс или разные модальности работы?
}

\author{
Максим С. Чистяков \\ Российская Федерация \\ E-mail: m_chistyakov@inbox.ru
}

Городской психоневрологический диспансер № 7 (со стационаром), г. Санкт-Петербург,

\begin{abstract}
Аннотация
Введение. В статье обращается внимание на различия межау психиатрическим и психотерапевтическим поАходами к Аиагностике и лечению психических расстройств. Подчеркивается, что эти поАХоАЫ имеют различающиеся преАставления о норме, разные цели и методы лечения. Этому часто не уделяется достаточного внимания в ^итературе и в клинической практике, что подчеркивает оригинальность статьи.
\end{abstract}

Теоретическое обоснование. Новым и оригинальным в статье является то, что при рассмотрении психиатрического поАхоАа и психотерапии в качестве разных и относительно независимых, но в то же время АОполняющих Аруг Аруга, модальностей работы с пациентами, автор статьи обращается к принципу Аополнительности, сорормулированному когАа-то Н. Бором в квантовой фризике Аля упорядочивания противоречивых Аанных, получаемых при разных условиях наблюдения и разных установках наблюдателя.

В статье приводится предлагаемая автором обобщенная схема психотерапевтического Аиагностического и мечебного процесса.

ОбсужАение и выводы. Опираясь на приведенную в предылущем разделе обобщенную схему психотерапевтического Аиагностического и лечебного процесса, автор показывает специфрические различия между диагностическим и лечебным процессом в психотерапии и психиатрии, что обычно недоучитывается в кАинической практике. Также в статье преАлагается при осуществлении психотерапии и при подготовке молодых психотерапевтов рассматривать три основных направления психотерапии (психодинамическое, гуманистическое, когнитивно-бихевиоральное) как Аополняющие Аруг Аруга, но в то же время как относительно независимые Аруг от Аруга целостные молели. Оригинальность статьи заключается также в утверждении, что в клинической практике, с точки зрения эфрорективности лечения, полезно рассматривать кажАого пациента в рамках и психиатрического по ххо $а$, и психотерапевтического подхода независимо и отАельно, периодически переключаясь от одной перспективы виАения к Аругой. 


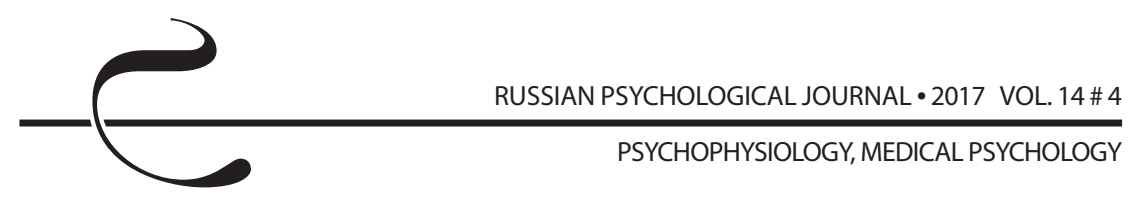

Заключение. Аелается вывоА, что преАставленный в статье новый поАХОА МОжет сАелать более ^огичной и ясной, свободной от противоречий и психиатрическую, медицинскую, и психотерапевтическую, сорокусированную на отношениях, модель. Он также может способствовать развитию кажАой из этих молелей.

\section{КАючевые слова}

психотерапия, психиатрия, нарушения функционирования психики, Аиагностическая система, нозологический поАхоА, паттерны отношений, модификация параметров отношений, модель процесса психотерапии, направления психотерапии, принцип Аополнительности

\section{Основные положения}

- психиатрический медицинский и психотерапевтический поАходы к лечению и Аиагностике психических расстройств качественно отличаются Аруг от Аруга с точки зрения понятия нормы, критериев Аиагностики, целей, методов и организации самого процесса ^ечения, а также с точки зрения терапевтической позиции помогающего специалиста;

- полезно Аля п^одотворного развития кажАой из этих моделей, а также Аля большей эфффективности ^ечения, с точки зрения клинической практики рассматривать эти Аве модели (психиатрическую медицинскую и психотерапевтическую, сорокусированную на паттернах отношений) как две относительно независимые, однако Аополняющие Аруг Аруга, в соответствии с когАа-то сорормулированным Н. Бором принципом Аополнительности;

- в процессе лечения пациентов и при подготовке молодых специалистов целесообразно рассматривать основные направления психотерапии (психодинамическое, когнитивно-поведенческое и гуманистическое) как относительно независимые, замкнутые, хотя и Аополняющие Аруг лруга теоретические молели, в соответствии с тем же принципом Аополнительности Н. Бора, не смешивая их в «еАиную экАектическую» модель;

- А^я большей эфффективности ^ечения в клинической практике, в соответствии с принципом Аополнительности Н. Бора, целесообразно кажАого отАельного пациента рассматривать в Авух отАельных, хотя и Аополняющих Аруг Аруга, системах коорАинат (с точки зрения психиатрического поАХОАа и с точки зрения психотерапевтического подхода), не смешивая эти перспективы виАения Аруг с Аругом.

\section{Для цитирования}

Чистяков М.С. Диагностический и лечебный процесс в психиатрии и психотерапии единый процесс или разные модальности работы? // Российский психологический журнал. - 2017. - Т. 14, № 4. - С. 162-179.

Материалы статьи получены 07.03.2017 


\title{
Diagnosis and Treatment in Psychiatry and Psychotherapy: A Unitary Process or Different Modes of Work?
}

\section{Maksim S. Chistyakov}

City Psychoneurological Dispensary no. 7 (with in-patient department), Saint-Petersburg, Russian Federation

E-mail: m_chistyakov@inbox.ru

\begin{abstract}
Introduction. The article draws attention to the difference between psychiatric and psychotherapeutic approaches to treating and diagnosing mental disorders. These approaches rely on different definitions of norm and also treatment goals and methods. Given the lack of critical attention paid to such a difference in the literature and clinical practice, this issue merits special attention. The paper presents a generalized model for the process of psychotherapeutic diagnosis and treatment.

Theoretical Basis. The study rested upon the principle of complementarity formulated by the physicist $\mathrm{N}$. Bohr in quantum mechanics for systematization of the data received in different circumstances of observation by the observers with different attitudes. Thus, the psychiatric approach and psychotherapy were considered not only as different and relatively independent but also as complementary modes of working with patients.

Discussion. The generalized model for the process of psychotherapeutic diagnosis and treatment demonstrated differences between diagnosis and treatment in psychotherapy and psychiatry. When practicing psychotherapy and training young psychotherapists, the three main kinds of psychotherapy (psychodynamic, humanistic, and cognitive behavioural) should be considered as complementary and also independent integral models. For effective treatment, clinicians must consider each patient separately within the psychiatric and psychotherapeutic approaches, switching from one perspective to another.
\end{abstract}

Conclusion. The new approach (a) can be readily used in practice to make the psychiatric and psychotherapeutic models more logical, clear, free from contradictions, and focused on the relationship and (b) contributes to the development of these models.

\section{Keywords}

psychotherapy, psychiatry, disorders of mental functioning, diagnostic system, nosological approach, relationship patterns, modification of relationship parameters, model of psychotherapy process, directions of psychotherapy, principle of complementarity 


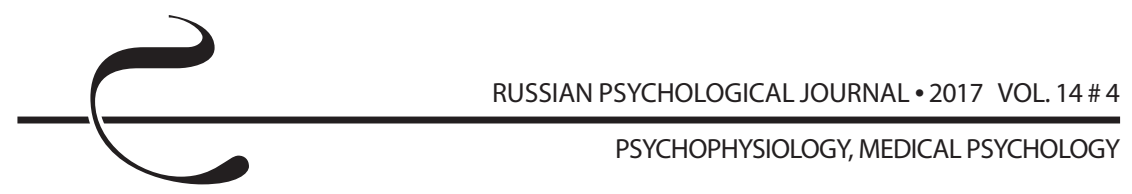

\section{Highlights}

- The psychiatric medical and psychotherapeutic approaches to treating and diagnosing mental disorders rely on qualitatively different definitions of norm, diagnosis criteria, goals and methods for the treatment process. They also differ in a specialist's therapeutic positioning.

- Following Niels Bohr's complementarity principle, the two models - psychiatric medical and psychotherapeutic (focused on relationship patterns) - should be considered as relatively independent but complementary. This is important for productive development of each of these models and for improving treatment efficiency.

- When treating patients and training young psychotherapists, the three main kinds of psychotherapy (psychodynamic, humanistic, and cognitive behavioural) should be considered as relatively independent, though complementary theoretical models. One should not mix them into a single eclectical model.

- For improving treatment efficiency, a clinician should consider each patient in independent and complementary coordinate systems - psychiatric and psychotherapeutic - not mixing these approaches.

\section{For citation}

Chistyakov M. S. Diagnosis and Treatment in Psychiatry and Psychotherapy: A Unitary Process or Different Modes of Work? Rossiiskii psikhologicheskii zhurnal - Russian Psychological Journal, 2017, V. 14, no. 4, pp. 162-179 (in Russian).

Original manuscript received 07.03.2017

\section{Введение}

Различия между психиатрическим и психотерапевтическим подходами к психическим расстройствам

Психиатрия и психотерапия смотрят на нарушения психического функционирования с разных позиций, имеют разные подходы, формируют разные классы понятий и используют разную терминологию, по-разному формулируют задачи диагностики и лечения, имеют несколько различающиеся представления о норме и патологии, опираются на разные концепции функционирования психики.

Под психотерапией автор статьи подразумевает, в соответствии с формулировкой, приведенной в «Психотерапевтической энциклопедии» под редакцией Б.Д. Карвасарского, «систему лечебного воздействия на психику и через психику на организм больного» [1, с. 448]. В нашей стране, в соответствии с действующим законодательством, к проведению психотерапии в полном объеме допускаются только врачи-психотерапевты, психологам разрешено «психологическое консультирование». Во многих других странах 
к проведению психотерапии в полном объеме помимо врачей допущены и психологи и социальные работники.

Психиатрия, как область медицины, опирается на биологическую, естественнонаучную основу. В медицине традиционно используется нозологический подход, подразумевающий представление о патологических процессах в человеческом организме, включая психику, как об определенных болезнях, имеющих определенные этиологию, патогенез, течение и исход. При этом специфичность клинической картины определенной болезни связана со специфическим сочетанием определенных синдромов, состоящих из отдельных симптомов $[2,3]$. Психотерапия и психологические концепции, на которые она опирается, используют совсем другой язык, имеют другое, чем психиатрия, представление о норме, имеют другие методы лечения и по-другому сформулированные цели лечения, и т. д. [4]. Хотя каждое из основных направлений психотерапии - психодинамическое, гуманистическое, когнитивно-бихевиоральное - использует свой собственный язык, по-своему формулирует цели лечения, использует разные методы в лечении [3, 4], основным инструментом лечения и осуществления изменений в психотерапии является определенным образом организованный процесс взаимодействия между пациентом и психотерапевтом. Какие бы методы психотерапевт ни использовал, в значительной степени [3], а по мнению ряда авторов - в основном $[5,6,7,8]$, агентом изменений в психотерапии являются отношения, которые устанавливаются между психотерапевтом и пациентом. Для ориентирования в процессе психотерапии в клинической практике необходима такая диагностическая система, которая описывает характеристики процесса взаимодействия между психотерапевтом и пациентом, увязывая их с характеристиками взаимоотношений пациента с другими людьми $[9,10]$, а не просто описывающая набор внутренних характеристик (в частности, симптомов, их сочетания в определенных синдромах) пациента как «вещи в себе», в качестве независимого от наблюдателя объекта наблюдения.

\section{Теоретическое обоснование}

\section{Принцип дополнительности}

Обратившись к истории науки, можно вспомнить когда-то введенный Нильсом Бором в квантовую физику принцип дополнительности, который был призван разрешить противоречия, связанные с разными картинами поведения объектов наблюдения этой науки (микрообъектов) в зависимости от условий наблюдения и установок наблюдателя [11]. Н. Бор, формулируя принцип дополнительности, просто предложил рассматривать разные теоретические модели, описывающие поведение этих объектов в разных условиях и при разных установках наблюдателя, как внутренне целостные, 
независимые друга от друга, и в то же время дополняющие друг друга в создании целостного представления о реальности [11, 12]. Формулировка принципа дополнительности напоминает о том, что перспективы видения одного объекта с разных сторон не противоречат, а дополняют друг друга в понимании того, что представляет из себя объект.

Принцип дополнительности и разные языки описания функционирования психики в норме и патологии

Возможно, можно было бы построить более связное и логичное понимание нормальных и патологических процессов в психике, если применить к различным формам и языкам описания функционирования психики в норме и патологии (в первую очередь, к психотерапевтическому (психоаналитическому) и психиатрическому подходам, но, кроме этого, и к подходам разных направлений психотерапии по отношению друг к другу) принцип дополнительности Н. Бора. Если бы мы рассматривали психотерапию (особенно психодинамическую психотерапию, как наиболее разработанную теоретическую модель) и психиатрию как имеющие свою внутреннюю логику, дополняющие друг друга, но в то же время отдельные, не перекрывающиеся друг с другом теоретические модели описания функционирования психики в норме и патологии, подобно тому, как в квантовой механике, например, концепция поведения электрона как волны дополняется концепцией поведения электрона как частицы, то не стало ли бы это таким же толчком в развитии нашего научного понимания психических явлений, каким послужило когда-то введение принципа дополнительности Н. Бора для понимания квантово-механических явлений? И если использовать психотерапевтическую (психодинамическую) и психиатрическую медицинскую модели как две независимые оси координат, через которые мы оцениваем каждого пациента, не обеспечило ли бы нам это большие ясность и понимание, чем попытки смешать психотерапию и психиатрию в одной модели? Психотерапия заинтересована в такой диагностической системе, в таких языке и формулировках, которые отражают типичные паттерны отношений, устанавливаемых пациентом с окружающими, включая врача, отражают возможную динамику разворачивающихся отношений с врачом. И для этих целей язык психиатрии порой слишком статичен, не отражает необходимую для психотерапии глубину понимания переживания пациентом его отношений с собой и другими [13].

В процессе практической работы для психотерапевта в плане диагностики важнее определить, является ли уровень организации личности (и, соответственно, уровень организации психологических защит) его пациента невротическим, пограничным или психотическим [14], чем точно 
сформулировать психиатрический диагноз в соответствии с МКБ-10. И если уровень организации личности пациента не психотический, то дальнейшие уточняющие диагностические формулировки, полезные для психотерапевтической работы, в психодинамической психотерапии, например, связаны не столько с уточнением психиатрического диагноза, с опорой на МКБ-10, сколько с определением привычных психологических защит пациента, наличия или отсутствия структурного дефекта эго (в случае нарциссических или пограничных пациентов) [15, 16], с определением основного внутриличностного бессознательного конфликта [17], с определением форм проявления всего вышеперечисленного в типичных паттернах взаимоотношений пациента с окружающими, с опорой на «треугольник Малана» (отношения со значимыми фигурами в прошлом - отношения с психотерапевтом в настоящем - отношения в настоящее время с другими людьми вне психотерапевтического кабинета) [18]. В когнитивно-бихевиоральной психотерапии, в свою очередь, такие уточняющие формулировки подразумевают выявление совокупности основных запускающих и подкрепляющих стимулов для проблемного поведения (паттернов поведения), определение основных когнитивных искажений, дисфункциональных интерпретативных схем, соотносящихся с проблемой пациента $[4,19]$. В гуманистической психотерапии уточняющие формулировки связаны с выявлением инконгруэнтности, отсутствия подлинности, уходов от контакта во взаимоотношениях между клиентом (пациентом) и психотерапевтом [4, 5, 20, 21] (с выявлением способов прерывания контакта пациентом на границе «организм - окружающая среда» - в гештальттерапии $[22,23])$.

\section{Общая модель процесса психотерапии}

Если в общем виде описать процесс психотерапии, то задачи психотерапевта любого направления - это, в соответствующей последовательности:

1. Превратить внутренний диалог пациента во внешний, с психотерапевтом [21], или прямо, как в психоанализе, экзистенциальной, когнитивной психотерапии, или опосредованно, в виде диалога пациента с воображаемыми образами внутренних объектов. В последнем случае такой диалог все равно отражает отщепленные аспекты диалога между пациентом и психотерапевтом, а образы внутренних объектов фактически являются переходными объектами в этом диалоге.

2. Создать безопасные условия для вовлечения пациента в этот развернутый вовне диалог, для развития отношений в этом диалоге с мобилизацией всех возникающих в его ходе чувств, при одновременном сохранении психотерапевтом баланса между эмпатически вовлеченной и наблюдающей (научной) позицией. 
3. Опираясь на наблюдение состояния определенных параметров этих развивающихся в диалоге отношений (между пациентом и психотерапевтом) в подходящие моменты, в соответствии с состоянием этих параметров в эти моменты, психотерапевт осуществляет интервенции с целью влияния на эти параметры и их модификации, отслеживая результаты интервенций по изменению состояния этих параметров. Те параметры отношений, на которых фокусируется внимание психотерапевта, будут отличаться в зависимости от направления психотерапии. В психоанализе этими параметрами будут сопротивления/психологические защиты [24], динамика отношений переноса/контрпереноса [9, 10, 25, 26, 27, 28, 29, 30, 31, 32]; в гуманистическом направлении - это способы ухода пациента от подлинного контакта с терапевтом в ситуации здесь-и-сейчас $[5,20]$ (способы прерывания цикла контакта - в гештальттерапии $[22,23])$, конгруэнтность/инконгруэнтность $[5,20]$; в когнитивно-бихевиоральной психотерапии - это проявляющиеся в виде автоматических мыслей когнитивные ошибки, иррациональные когнитивные убеждения, а также совокупность запускающих и подкрепляющих стимулов для проблемного поведения пациента $[3,4,19]$. Под влиянием интервенций психотерапевта и модификации этих параметров (в случае успешной психотерапии) отношения в диалоге развиваются и меняются в более конструктивную сторону [21].

4. Психотерапевт способствует сепарации в отношениях пациента с ним (с точки зрения психоанализа, происходит разрешение «невроза переноса» $[25,27])$. Одновременно происходит интернализация этих модифицированных в диалоге с психотерапевтом отношений (трансмутирующая интернализация, Х. Кохут [6]), в результате чего изменяются в более конструктивную сторону отношения пациента с самим собой (с точки зрения психоанализа, меняются психические структуры эго, производные от объектных отношений - внутренние репрезентации самости (self) и объекта, модифицируется супер-эго) [30], меняется ощущение своего Я (происходят изменения в ощущении своей идентичности, с точки зрения Э. Эриксона [33], изменяется ощущение, переживание самости (self), с точки зрения X. Кохута [6], меняются отношения пациента с другими людьми и окружающим миром. Это то, что в стратегической психотерапии называется изменениями второго порядка [34]. Происходит завершение психотерапии.

Эта схема работает в том числе и в когнитивно-бихевиоральной психотерапии, что подтверждается исследованиями, продемонстрировавшими, что наиболее действенными интервенциями психотерапевтов в вызывании положительных изменений у пациентов в когнитивно-бихевиоральной психотерапии оказались те, которые были связаны с фокусом на происходившем в отношениях между пациентом и психотерапевтом [35, 36, 37]. 


\section{Обсуждение и выводы}

Различия между психотерапевтическим и психиатрическим диагностическим и лечебным прочессами

Если обратиться к вышеприведенной схеме, то можно заметить, что на первом этапе взаимодействия с пациентом в беседе и для психиатра и для психотерапевта важно создать условия для того, чтобы пациент раскрыл свои переживания, вовлечь пациента во внешний диалог. И здесь могут быть некоторые сходства в работе. Но уже со следующего этапа появляются существенные различия. Для психиатра стоит задача вычленить из переживаний пациента, проявляющихся во взаимодействии с ним, информацию, значимую для отнесения пациента как объекта и носителя определенных симптомов к какой-то определенной диагностической категории классификации заболеваний с целью подбора биологического лечения. При этом он отбрасывает все (определенные жалобы, переживания, установки, особенности поведения, речи), что не соответствует этой цели. И здесь для психиатра важно максимально сохранить позицию научного наблюдателя для обеспечения максимальной объективности. Слишком большая эмоциональная вовлеченность в беседу, как со стороны пациента, так и со стороны психиатра, может помешать выполнению этой задачи. Соответственно, беседу важно выстраивать так, чтобы эта вовлеченность была на минимально необходимом (для раскрытия пациентом своих переживаний) уровне.

Для психотерапевта стоит задача создать безопасные условия для максимального (в том числе эмоционального) вовлечения пациента во внешний диалог. Только таким образом психотерапевт может наиболее эффективно выполнить следующие задачи. А именно: определив для целей диагностики уровень организации личности пациента (невротический, пограничный или психотический), выявлять, отслеживать и оценивать определенные параметры развивающегося во всей полноте переживаний внешнего диалога, модифицировать эти параметры с помощью определенных интервенций и отслеживать изменения. И для психотерапевта для выполнения этих задач важно постоянно сохранять баланс между эмпатической позицией (позицией эмоционального вовлечения) и позицией научного наблюдателя. Как видно из всего этого, после первого этапа во взаимодействии с пациентом задачи, организация процесса, необходимый фокус внимания, сам язык описания процесса и даже необходимая позиция в диалоге с пациентом (баланс между эмпатической эмоциональной вовлеченностью и научной наблюдающей позицией) для психотерапевта и психиатра сильно различаются. И для психотерапевта важны очень специфические навыки, которыми он отчасти овладевает в том числе и через свою личную психотерапию, чтобы он мог эффективно выполнять свои задачи. Из этого следует неоправданность 
иногда встречающихся среди психиатров, не получивших достаточной специальной подготовки в области психотерапии, утверждений, что они в своей психиатрической работе «тоже осуществляют психотерапию». Все это означает, что психотерапия подразумевает особую модальность процесса, качественно отличающуюся от модальности работы психиатра.

\section{Принцип дополнительности и использование подходов разных на- правлений психотерапии}

Если обратиться к применению принципа дополнительности внутри области психотерапии к теоретическим моделям и подходам разных направлений психотерапии и их отношениям друг с другом, то можно вспомнить, что в нашей стране является достаточно принятым, особенно в области государственной подготовки психотерапевтов, использование так называемого эклектического подхода в психотерапии. Под этим подразумевается, что психотерапевт осваивает самые разные методы психотерапии и применяет те или иные из этих методов в зависимости от того, где и когда это подходит тому или другому пациенту. Однако при таком подходе могут возникнуть некоторые сложности. Опять же, из вышеприведенной схемы психотерапевтического процесса видно, что на этапе модификации отношений в диалоге и отслеживания соответствующих изменений каждое направление психотерапии фокусируется на своих параметрах, использует свои, особые, интервенции для изменения этих параметров, по-разному организует этот процесс модификации (изменения), использует свой особый язык для описания этого процесса. Каждое направление, готовя психотерапевтов, обучает их не только теоретически, но и на их собственном опыте, при прохождении ими личной психотерапии в определенном объеме, отслеживать эти параметры и находить способы, влияющие на них. И личная психотерапия помогает психотерапевту не только не путать свой личный жизненный опыт с опытом его пациентов в процессе работы с ними, с точки зрения отслеживания определенных параметров отношений, но и помогает научиться лучше использовать свою интуицию в этом модифицирующем процессе при работе с его пациентами.

Возвращаясь к упомянутой выше эклектической модели, во всяком случае в таком виде, в каком она рассматривается в отечественной психотерапии, представляется сомнительным, что психотерапевт может на самом главном, модифицирующем этапе психотерапии быть достаточно эффективным, пытаясь одновременно отслеживать самые разные параметры отношений, специфичные для каждого направления психотерапии, будет одновременно соблюдать все, разные для разных направлений, условия организации этого процесса модификации, способствующие выявлению и изменению специфических 
параметров, будет осуществлять все возможные в разных направлениях психотерапии интервенции, без создания и соблюдения условий (создания почвы), специфичных для каждого направления, являющихся необходимой основой для того, чтобы данная интервенция была возможна и эффективна. Вместо этого, при таком понимании эклектичности, скорее всего представлениям психотерапевта будет не хватать связности и структурированности, его действия будут хаотичными, психотерапия - поверхностной, и психотерапевт будет часто прибегать к помощи медикаментов. Поэтому автору представляется, что более эффективным является подход, рассматривающий разные направления и формы психотерапии как дополнительные друг к другу, но при этом отдельные, внутренне замкнутые и структурированные модели (системы), в соответствии с тем, как это сформулировано в принципе дополнительности Н. Бора $[11,12]$.

Соответственно, более эффективной представляется подготовка психотерапевтов, осуществляемая по каждому направлению психотерапии (по психодинамическому, гуманистическому, когнитивно-бихевиоральному) отдельно, как это происходит за рубежом. Тогда это будут специалисты в определенном направлении психотерапии, со специфическим мышлением, но и с хорошо отработанными навыками в этом направлении. Безусловно, узкие специалисты в данном направлении должны неплохо знать и другие направления психотерапии, при необходимости заимствовать какие-то интервенции и техники из других направлений. Но у них должна быть определенная система взглядов, связанная с данным определенным направлением психотерапии, определяющая стратегию и организацию работы с пациентами. Только воспринятые через призму такой системы взглядов, эти заимствованные из других направлений техники и могут быть интегрированы в работу психотерапевта наиболее эффективным образом.

\section{Модальности психотерапевтической работы и работы психиатра в клинической практике}

Для автора остается открытым вопрос, влияет ли на качество, глубину психотерапевтического процесса осуществление и психотерапевтической и психиатрической помощи пациенту одним, подготовленным в обеих этих областях, специалистом [38, 39], хотя и у автора и у некоторых его коллег имеется положительный опыт такой работы, с неплохими результатами. Однако, в любом случае, если и ту и другую помощь пациенту оказывает один специалист, по мнению и опыту автора, полезнее для качества лечебного процесса, чтобы специалист работал, рассматривая пациента в каждой из двух этих модальностей (психиатрической и психотерапевтической) независимо и отдельно, при необходимости периодически переключаясь 
от одной перспективы видения к другой, а не смешивал психиатрическую и психотерапевтическую модели в один конгломерат. Либо в качестве психиатра и психотерапевта для пациента могут выступать два разных специалиста. По мнению автора, при использовании любой формы психотерапии в комплексном лечении больных, у которых периодически повторяются психотические состояния, участие в лечении двух разных специалистов, психиатра и психотерапевта (вне зависимости от наличия у последнего психиатрической подготовки), является обязательным условием (в силу разной модальности работы психиатра и психотерапевта).

\section{Заключение}

Таким образом, введенный Н. Бором в начале XX в. принцип дополнительности, который был призван вернуть внутреннюю логику противоречивой картине, возникающей при наблюдении квантово-механического мира, может помочь и в попытках прояснить, сделать более внутренне логичным и связным наше видение психических процессов с разных перспектив, в норме и патологии. Рассмотрение психотерапевтической (особенно психодинамической психотерапевтической) модели, сфокусированной на отношениях, как отдельной и дополнительной к психиатрической, медицинской модели, сфокусированной на пациенте как на объекте, обладающем определенными свойствами (симптомами), может помочь очистить от противоречий, сделать более внутренне логичной, ясной каждую из этих моделей. В то же время, такой взгляд может облегчить и восприятие каждой из этих моделей как дополняющих друг друга, а не конкурирующих друг с другом, равнозначных, а не находящихся в отношениях подчинения [38, 39]. По мнению автора, такой взгляд может способствовать как развитию каждой из этих моделей, так и их обогащающему влиянию друг на друга. Также принцип дополнительности может быть полезен и внутри области психотерапии. В частности, разные направления психотерапии с их разным языком, разным фокусом в психотерапевтической работе тоже полезно рассматривать как дополнительные друг к другу, и в то же время отдельные, проводя подготовку молодых специалистов по каждому направлению психотерапии отдельно.

Также, с точки зрения клинической практики, может быть полезно, используя принцип дополнительности, рассматривать каждый случай психического расстройства одновременно и в то же время отдельно в двух параллельных плоскостях (в двух системах координат), с точки зрения медицинской, психиатрической модели, и с точки зрения психотерапевтической (в частности, психодинамической) модели. 


\section{Литература}

1. Психотерапевтическая энциклопедия / под ред. Б. Д. Карвасарского. СПб. : Питер, 2006. - 3-е изд. - 946 с.

2. Психиатрия. Руководство для врачей в двух томах / под ред. акад. РАМН А. С. Тиганова. - М. : Медицина, 2012. - Т. 1. - 807 с.

3. Wampold B. E., Imel Z. E. The Great Psychotherapy Debate: The Evidence for What Makes Psychotherapy Work. - New York, London : Routledge, 2015. - $334 \mathrm{p}$.

4. Прохазка Дж., Норкросс Дж. Системы психотерапии. - СПб. : ПраймЕврознак, 2007. - 384 с.

5. Бьюдженталь Д. Искусство психотерапевта. - СПб. : Корвет, 2011. - 316 с.

6. Кохут Х. Восстановление самости. - М. : Когито-Центр, 2017. - 320 с.

7. Столороу Р., Брандшафт Б., Атвуд Д. Клинический психоанализ. Интерсубъективный подход. - М. : Когито-Центр, 2011. - 256 с.

8. Ялом И. Теория и практика групповой психотерапии. - СПб. : Питер, 2000. -640 c.

9. Caligor E., Kernberg O. F., Clarkin J. Handbook of Dynamic Psychotherapy for Higher Level Personality Pathology. - Arlington : American Psychiatric Publishing, 2007. - 284 p.

10. Clarkin J. F., Yeomans F. E., Kernberg O. F. Transference-Focused Psychotherapy for Borderline Personality Disorder: A Clinical Guide. - Arlington : American Psychiatric Publishing, 2015. - 427 p.

11. Бор Н. Квант действия и описание природы // Избранные труды : в 2 томах. - М. : Наука, 1971. - Т. 2. - С. 56-61.

12. Бор Н. Квантовая физика и философия // Избранные научные труды : в 2 томах. - M. : Наука, 1971. - T. 2. - C. 526-532. - DOI: 10.3367/ UFNr.0067.195901c.0037

13. Решетников М. М. Современная психотерапия: медицинская и психологическая модели // Вестник психотерапии. - 2016. - № 60 (65). C. 7-16.

14. Мак-Вильямс Н. Психоаналитическая диагностика: понимание структуры личности в клиническом процессе. - М. : Класс, 2015. - 592 с.

15. Kernberg O. F. Borderline Conditions and Pathological Narcissism. - New York : Jason Aronson, 1975. - 376 p.

16. Kernberg O. Borderline Personality Organization // Essential papers on borderline disorders / M. H. Stone (ed.). - New York: New York University Press, 1986. - P. 279-320. - DOI: 10.1177/000306516701500309

17. Фрейд 3. Введение в психоанализ : Лекции. - М. : Наука, 1989. - 456 с.

18. Malan D. H. Toward the Validation of Dynamic Psychotherapy: A Replication. - New York : Plenum Medical Books, 1976. - 298 p. 
19. Бек Д. Когнитивная терапия. Полное руководство. - М. : ДиалектикаВильямс, 2017. -400 с.

20. Роджерс К. Клиент-центрированная психотерапия: теория, практика и применение. - М. : Психотерапия, 2007. - 560 с.

21. Сименс X. Практическое руководство для Гештальттерапевтов. - СПб. : Издательство Пирожкова, 2008. - 168 с.

22. Перлз Ф., Хеффферлин Р., Гудмэн П. Опыты психологии самопознания (практикум по гештальттерапии). - М. : Гиль-Эстель, 1993. - 240 с.

23. Зинкер Д. В поисках хорошей формы. Гештальт-терапия с супружескими парами и семьями. - Санкт-Петербург : Корвет, 2015. - 272 с.

24. Strean H. S. Resolving resistances in psychotherapy. - New York : Brunner Mazel, 1990. - $316 \mathrm{p}$.

25. Гринсон Р. Техника и практика психоанализа. - М. : Когито-Центр, 2010. $480 \mathrm{c.}$

26. Bird B. Notes on transference: universal phenomenon and hardest part of analysis // Essential papers on transference / A. H. Esman (ed.). - New York: New York University Press, 1990. - P. 331-361. - DOI: 10.1177/000306517202000203

27. Freud S. The Dynamics of transference // Essential papers on transference / A. H. Esman (ed.). - New York : New York University Press, 1990. - P. 28-36.

28. Greenacre $P$. The role of transference: practical considerations in relation to psychoanalytic therapy // Essential papers on transference / A. H. Esman (ed.). - New York: New York University Press, 1990. - P. 124-135. - DOI: 10.1177/000306515400200406

29. Kernberg O. An ego-psychology-object relations theory approach to the transference // Essential papers on transference / A. H. Esman (ed.). - New York: New York University Press, 1990. - P. 492-510.

30. Kernberg O. Structural derivatives of object relations // Essential papers on object relations / P. J. Buckley (ed.). - New York : New York University Press, 1986. - P. 309-342.

31. Stone $L$. The transference-countertransference complex // Essential papers on transference / B. Wolstein (ed.). - New York : New York University Press, 1988. - P. 270-281.

32. Winnicott D. W. Counter-transference // Essential papers on transference / B. Wolstein (ed.). - New York : New York University Press, 1988. - P. 262-269. DOI: $10.1111 / \mathrm{j} .2044-8341.1960 . t b 01220 . x$

33. Эриксон Э. Детство и общество. - СПб. : Ленато , АСТ , Фонд «Университетская книга», 1996. - 592 с.

34. Нардонэ Д., Вачлавик П. Искусство быстрых изменений. Краткосрочная стратегическая терапия. - М. : Изд-во Института психотерапии, 2006. 195 c. 
35. Jones E. E., Pulos S. M. Comparing the Process in Psychodynamic and Cognitive-Behavioral Therapies // Journal of Consulting and Clinical Psychology. - 1993. - Vol. 61, № 2. - P. 306-316. - DOI: 10.1037//0022-006X.61.2.306

36. Milton J. Psychoanalysis and cognitive behaviour therapy - rival paradigms or common ground? //The International Journal of Psychoanalysis. - 2001. Vol. 82, Issue 3. - P. 431-447. - DOI: 10.1516/DVLN-RK5E-C1YV-ME4V

37. Wiser S., Goldfried M. Verbal interventions in significant psychodynamicinterpersonal and cognitive-behavioral therapy sessions // Psychotherapy Research. - 1996. - № 6. - Р. 309-319.

38. Бобров А. Е. Методологические аспекты изучения психосоматических соотношений и перспективы интегративной медицины // Психосоматическая медицина - 2016 : труды XI международного конгресса. - СПб. : Человек, 2016. - С. 6-9.

39. Курпатов В. И., Гладышенко А. В. Кризис или коллапс российской психотерапии? // Психосоматическая медицина - 2016 : труды XI международного конгресса. - СПб. : Человек, 2016. - С. 33-37.

\section{References}

1. Karvasarskiy B. D. (ed.) Psikhoterapevticheskaya entsiklopediya [Psychother apeutic encyclopedia]. St. Petersburg, Piter Publ., 2006. 946 p.

2. Tiganov A. S. (ed.) Psikhiatriya. Rukovodstvo dlya vrachei v dvukh tomakh [Psychiatry: A guide for physicians in two volumes]. Moscow, Meditsina Publ., 2012, V. 1. 807 p.

3. Wampold B. E., Imel Z. E. The great psychotherapy debate: The evidence for what makes psychotherapy work. New York, London, Routledge, 2015. 334 p.

4. Prokhazka Dzh., Norkross Dzh. Sistemy psikhoterapii [Psychotherapy systems]. St. Petersburg, Praim-Evroznak Publ., 2007. 384 p.

5. Bugental, J. F. T. The art of the psychotherapist (Russ. ed.: B'yudzhental' D. Iskusstvo psikhoterapevta. St. Petersburg, Korvet Publ., 2011. 316 p.).

6. Kohut $\mathrm{H}$. The restoration of the self (Russ. ed.: Kokhut Kh. Vosstanovlenie samosti. Moscow, Kogito-Tsentr Publ., 2017. 320 p.).

7. Stolorow, R. D., Brandchaft, B., \& Atwood, G. E. Psychoanalytic treatment: An intersubjective approach (Russ. ed.: Stolorou R., Brandshaft B., Atvud D. Klinicheskii psikhoanaliz. Intersub"ektivnyi podkhod. Moscow, Kogito-Tsentr Publ., 2011. 256 p.).

8. Yalom I. D. The theory and practice of group psychotherapy (Russ. ed.: Yalom I. Teoriya i praktika gruppovoi psikhoterapii. St.Petersburg, Piter Publ., 2000.640 p.).

9. Caligor E., Kernberg O. F., Clarkin J. Handbook of dynamic psychotherapy for higher level personality pathology. Arlington, American Psychiatric Publishing, 2007. 284 p. 
10. Clarkin J. F., Yeomans F. E., Kernberg O. F. Transference-focused psychotherapy for borderline personality disorder: A clinical guide. Arlington, American Psychiatric Publishing, 2015. 427 p.

11. Bohr N. The quantum of action and the description of nature (Russ. ed.: Bor N. Kvant deistviya iopisanie prirody. Izbrannye trudy: 2 tomakh. Moscow, Nauka Publ., 1971, V. 2, pp. 56-61).

12. Bohr N. Quantum physics and philosophy (Russ. ed.: Bor N. Kvantovaya fizika i filosofiya. Izbrannye nauchnye trudy: 2 tomakh. Moscow, Nauka Publ., 1971. V. 2, pp. 526-532. DOI: 10.3367/UFNr.0067.195901c.0037).

13. Reshetnikov M. M. Contemporary psychotherapy: Medical and psychological model. Vestnik psikhoterapii - Bulletin of Psychotherapy, 2016, no. 60 (65), pp. 7-16 (in Russian).

14. Mack-Williams N. Psychoanalytic diagnosis: understanding personality structure in the clinical process (Russ. ed.: Mak-Vil'yams N. Psikhoanaliticheskaya diagnostika: ponimanie struktury lichnosti v klinicheskom protsesse. Moscow, Klass Publ., 2015. 592 p.).

15. Kernberg O. F. Borderline conditions and pathological narcissism. New York, Jason Aronson, 1975. $376 \mathrm{p}$.

16. Kernberg O. Borderline personality organization. In: M. H. Stone (ed.) Essential papers on borderline disorders. New York, New York University Press, 1986, pp. 279-320. DOI: $10.1177 / 000306516701500309$

17. Freud Z. Introductory lectures on psychoanalysis (Russ. ed.: Freid Z. Vvedenie v psikhoanaliz: Lektsii. Moscow, Nauka Publ., 1989. 456 p.).

18. Malan D. H. Toward the validation of dynamic psychotherapy: a replication. New York, Plenum Medical Books, 1976. 298 p.

19. Beck J. Cognitive therapy: Basics and beyond (Russ. ed.: Kognitivnaya terapiya. Polnoe rukovodstvo. Moscow, Dialektika-Vil'yams Publ., 2017. 400 p.).

20. Rogers C. R. Client-centered therapy: Its current practice, implications and theory (Russ. ed.: Klient-tsentrirovannaya psikhoterapiya: teoriya, praktika i primenenie. Moscow, Psikhoterapiya Publ., 2007. 560 p.).

21. Siemens H. Guidance for Gestalt therapists (Russ. ed.: Prakticheskoe rukovodstvo dlya Geshtal'tterapevtov. St.Petersburg, Izdatel'stvo Pirozhkova Publ., 2008. 168 p.).

22. Perls F., Hefferline R., Goodman P. Gestalt therapy: Excitement and growth in the human personality (Russ. ed.: Perlz F., Khefferlin R., Gudmen P. Opyty psikhologii samopoznaniya (praktikum po geshtal'tterapii). Moscow, Gil'-Estel' Publ., 1993. 240 p.).

23. Zinker, J. (1994). In search of good form: Gestalt therapy with couples and families (Russ. ed.: Zinker D. V poiskakh khoroshei formy. Geshtal't-terapiya s supruzheskimi parami i sem'yami. St. Petersburg, Korvet Publ., 2015. 272 p.). 
24. Strean H. S. Resolving resistances in psychotherapy. New York, Brunner Mazel, 1990. 316 p.

25. Greenson R. The techniques and practice of psychoanalysis (Russ. ed.: Grinson R. Tekhnika i praktika psikhoanaliza. Moscow, Kogito-Tsentr Publ., 2010.480 p.).

26. Bird B. Notes on transference: universal phenomenon and hardest part of analysis. In: A. H. Esman (ed.) Essential papers on transference. New York, New York University Press, 1990, pp. 331-361.DOI: 10.1177/000306517202000203

27. Freud S. The Dynamics of transference. In: A. H. Esman (ed.) Essential papers on transference. New York, New York University Press, 1990, pp. 28-36.

28. Greenacre P. The role of transference: practical considerations in relation to psychoanalytic therapy. In: A. H. Esman (ed.) Essential papers on transference. New York. New York University Press, 1990, pp. 124-135. DOI: 10.1177/000306515400200406

29. Kernberg O. An ego-psychology-object relations theory approach to the transference. In: A. H. Esman (ed.) Essential papers on transference. New York, New York University Press, 1990, pp. 492-510.

30. Kernberg O. Structural derivatives of object relations. In: P. J. Buckley (ed.) Essential papers on object relations. New York, New York University Press, 1986, pp. 309-342.

31. Stone L. The transference-countertransference complex. In: B. Wolstein (ed.) Essential papers on transference. New York, New York University Press, 1988, pp. 270-281.

32. Winnicott D. W. Counter-transference. In: B. Wolstein (ed.) Essential papers on transference. New York, New York University Press, 1988, pp. 262-269. DOI: 10.1111/j.2044-8341.1960.tb01220.x

33. Erikson E. Childhood and society (Russ. ed.: Erikson E. Detstvo i obshchestvo. St. Petersburg, Universitetskaya kniga Publ., 1996. 592 p.).

34. Nardone, D., Waclawik P. The art of rapid change. Short-term strategic therapy (Russ. ed.: Nardone D., Vatslavik P. Iskusstvo bystrykh izmenenii. Kratkosrochnaya strategicheskaya terapiya. Moscow, Institute of Psychotherapy Publ., 2006. 195 p.).

35. Jones E. E., Pulos S. M. Comparing the process in psychodynamic and cognitive-behavioral therapies. Journal of Consulting and Clinical Psychology, 1993, V. 61, no. 2, pp. 306-316. DOI: 10.1037//0022-006X.61.2.306

36. Milton J. Psychoanalysis and cognitive behaviour therapy - rival paradigms or common ground? The International Journal of Psychoanalysis, 2001, V. 82, Issue 3, pp. 431-447. DOI: 10.1516/DVLN-RK5E-C1YV-ME4V

37. Wiser S., Goldfried M. Verbal interventions in significant psychodynamicinterpersonal and cognitive-behavioral therapy sessions. Psychotherapy Research, 1996, no. 6, pp. 309-319. 
38. Bobrov A. E. Metodologicheskie aspekty izucheniya psikhosomaticheskikh sootnoshenii i perspektivy integrativnoi meditsiny [Methodological aspects of studying psychosomatic interrelations and the future of integrative medicine]. Trudy XI mezhdunarodnogo kongressa: Psikhosomaticheskaya meditsina - 2016 [Proc. the 11th International Congress "Psychosomatic medicine 2016"]. St. Petersburg, Chelovek Publ., 2016, pp. 6-9.

39. Kurpatov V. I., Gladyshenko A. V. Krizis ili kollaps rossiiskoi psikhoterapii? [Russian psychotherapy: crisis vs. collapse]. Trudy XI mezhdunarodnogo kongressa: Psikhosomaticheskaya meditsina - 2016 [Proc. the 11 th International Congress "Psychosomatic medicine 2016"]. St. Petersburg, Chelovek Publ., 2016, pp. 33-37. 